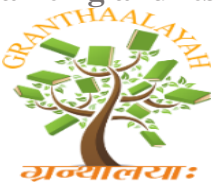

Arts

\section{INTERNATIONAL JOURNAL OF RESEARCH GRANTHAALAYAH A knowledge Repository}

DOI: https://doi.org/10.29121/granthaalayah.v7.i11.2019.3704

\title{
COLOUR SPECTRUM
}

\author{
Dr. Asha Gaikwad *1 \\ ${ }^{* 1}$ Phisics Department, Shaskiya Maharani Laxmi Bai, Kila Maidan, Indore (M.P.)
}

\begin{abstract}
Colour is one of the most important elements in our life. Colour can attract our attention and change our mood. When white light dispersed by prism or a diffraction grating the colours are produce. There is a continuous change in wavelength from red to violet. Seven colours are usually distinguished - Violet, Indigo, Blue, Green Yellow, Orange and Red. It is called spectrum. A rainbow shows the colours of the spectrum. It is a range of Light waves or radio waves within particular frequencies.
\end{abstract}

Keywords: Colour; Spectrum.

Cite This Article: Dr. Asha Gaikwad. (2019). "COLOUR SPECTRUM." International Journal of Research - Granthaalayah, 7(11SE), 80-82. 10.29121/granthaalayah.v7.i11.2019.3704.

\section{Introduction}

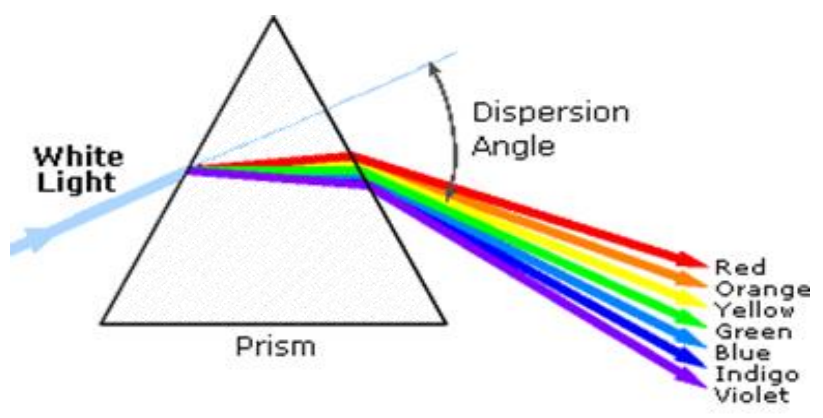

\section{Spectroscopy}

Spectroscopy is a very important tool in astronomy. Light is a energy that moves through space. Light has many different frequencies. Spectrometers are instruments which is spread light out into its wavelengths creating a spectra. In the spectra emission and absorption lines found which are the characteristics of atoms and molecules. An emmission line occurs when an electron drops down to a lower orbit around the nucleus of an atom and looses energy. An absorption line occurs when electrons moves to a higher orbit by absorbing energy. Each atom has a unique spacing of orbits. Each atom can emit or absorb only certain energies or wavelengths. So the spectral line is unique for each atom. 


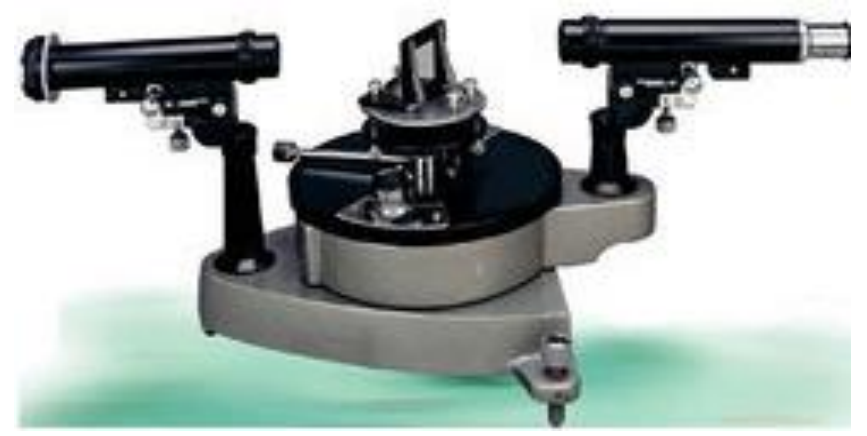

There are Three types of Spectra - Contunuous Spectra, Emission Spectra and Absorption spectra.

\section{Continuous Spectra}

It also called a thermal or black body spectra. It is emmitted by an object that rediates heat. The light is spread out into a continuous band whit every wavelength having some amount of radiation. When Sunlight is passed through a prism it spread in different colours. A blackbord is a body that absorbs all electromegnatic radiation incident on it. By the second law of thermo dynamics a body always tries to stay in a thermal equilibrium. To stay in thermal equilibrium a black body must emit radiation at the same rate as it absorbs. So it must also be a good emitter of radition. Emmiting electromagnetic waves of many frequencies.

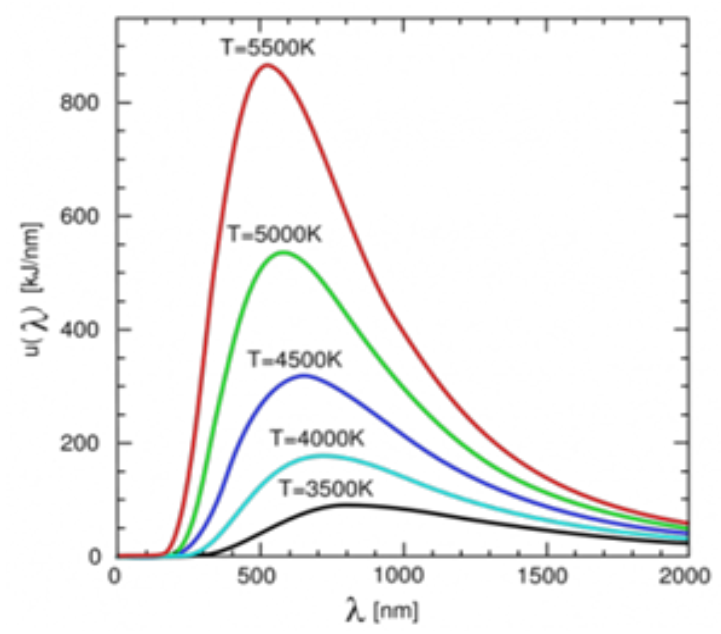

\section{Absorption Spectra}

In some spectrum we get the presence of dark lines. The lines are cause by the Sun's atmosphere absorbing light at certain wavelength cause in the intensity of the light at this wavelength to draw and appear it dark. The atoms and molecules in a gas will absorb only certain wavelength of light. The pattern of these lines is unique to each elements. The absorption spectrum found from reasons in space where a cooler gas lies between us and a hot source. The absorption spectra produces from stars, planets with atmospre and galaxcies.

Emission Spectra- 


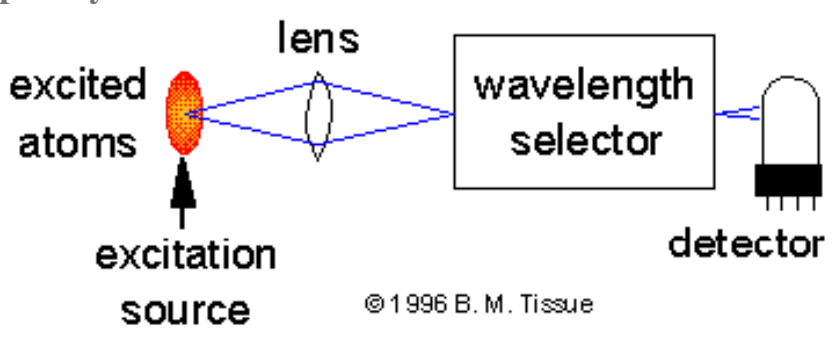

An Emission Spectra occurs when the atoms and molecules in a hot gas emit extra light at certain wavelengths. The pattern of these lines is unique for each element. We can see Emission Spectra from comets, Nebula and certain types of stars.

\section{Emission Spectrum of Hydrogen Atom}

In the Emission Spectrum of Hydrogen Atom mainly the spectral lines of the following series are obtained - Lyman Series, Balmer Series, Paschen Series, Brakett Series, Pfund Series.

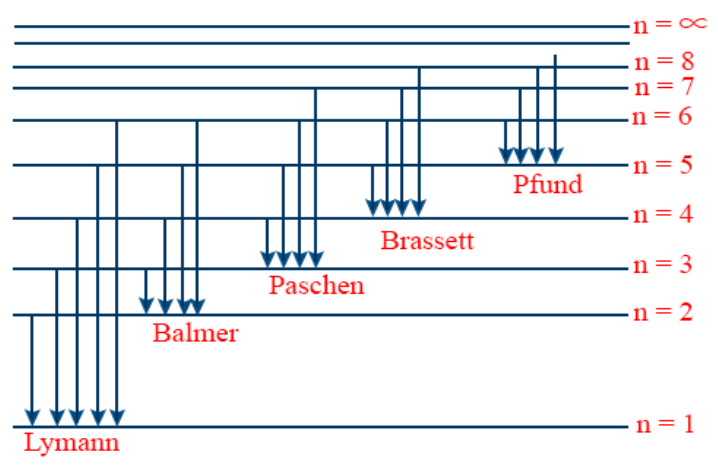

- Lyman Series - If the transiction of electron is from any higher orbit to the first orbit, the spectral lines of lyman series are obtained in the ultraviolet reason.

- Balmer Series - If the transition of electron is from any higher orbit to the second orbit, the spectral lines of Balmer series are obtained in the visible reason.

- Paschen Series - If the transition of electron is from any higher orbit to the third orbit, the spectral lines of Paschan Series are obtained in the infrared reason.

- Brackett Series - If the transition of electron is from any higher orbit to the fourth orbit the spectral lines of bracket series are obtained in the far infrared reason.

- Pfund Series - If the transition of electron is from any higher orbit to the fifth orbit, the spectral lines of Pfund Series are obtained in the far far infrared reason.

In these way we can define different types of spectrums. 